\title{
EXCISION OF THE ACROMION IN TREATMENT OF THE SUPRASPINATUS SYNDROME
}

\author{
Report of Ninety-five Excisions * \\ J. R. Armstrong, London, England
}

The majority of patients who complain of a painful shoulder are suffering either from periarthritis, "frozen shoulder," or from a supraspinatus lesion. Lesions of the supraspinatus tendon at or close to its insertion, where it is intimately associated with the subacromial bursa, give rise to a characteristic combination of symptoms-the supraspinatus syndrome - which are mechanical in origin. In the middle range of abduction movement the tendon impinges on the overlying processes and the tendon and bursa are compressed between the humerus and acromion (Fig. 1). When there is abnormality of the tendon or bursa this pressure causes pain, and any movement which tends to bring the lesion into contact with the acromion causes reflex muscle spasm. The supraspinatus syndrome is usually characteristic, although in its later stages it may be complicated by true limitation of shoulder movement, due partly to disuse and partly to adhesion formation in the region of the subacromial bursa, which masks the typical symptoms and complicates both diagnosis and treatment.

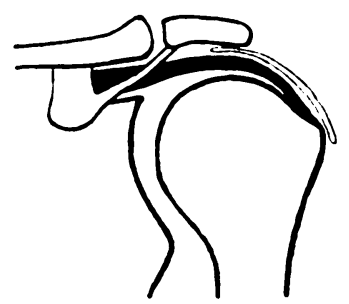

In the middle range of abduction the supraspinatus tendon and subacromial bursa are com pressed between the upper end of the humerus and the acromion.

The results of conservative treatment-Most patients with the supraspinatus syndrome recover either spontaneously or after conservative treatment. Many measures have been advocated and good results have been reported with each of them. It is probably true that about 90 per cent. of patients with this condition get well in a few weeks. In the remaining 10 per cent., symptoms persist stubbornly for many months in spite of such treatment as rest, physiotherapy, manipulation, active exercises, infiltration with local anaesthetic or deep X-ray therapy, and it is in this group of cases that excision of the acromion is indicated.

\section{THE AIMS AND PRINCIPLES OF EXCISION OF THE ACROMION}

The principle underlying excision of the acromion is simple. Intermittent pressure associated with abduction or forward flexion of the arm causes pain and muscle spasm, and constantly repeated irritation prevents healing of the lesion. Excision of the acromion relieves this pressure. A full range of painless shoulder movement then becomes possible and most of the symptoms disappear by the time the patient has recovered from the immediate effects of the operation. When relieved of irritation, the underlying condition slowly resolves or heals.

* Based on a paper read at the Annual Meting of the British Orthopaedic Association in Belfast, October 1948 
To ensure successful results a considerable amount of bone must be removed. This point was not at first appreciated and in the first nine cases in this series not enough of the acromion was excised; in attempting to preserve the acromio-clavicular articulation the bone was divided immediately lateral to the joint (Fig. 3). At operation there appeared to be adequate clearance but the results were unsatisfactory in five of these patients. The upper end of the humerus lies slightly anterior to, rather than immediately below, the

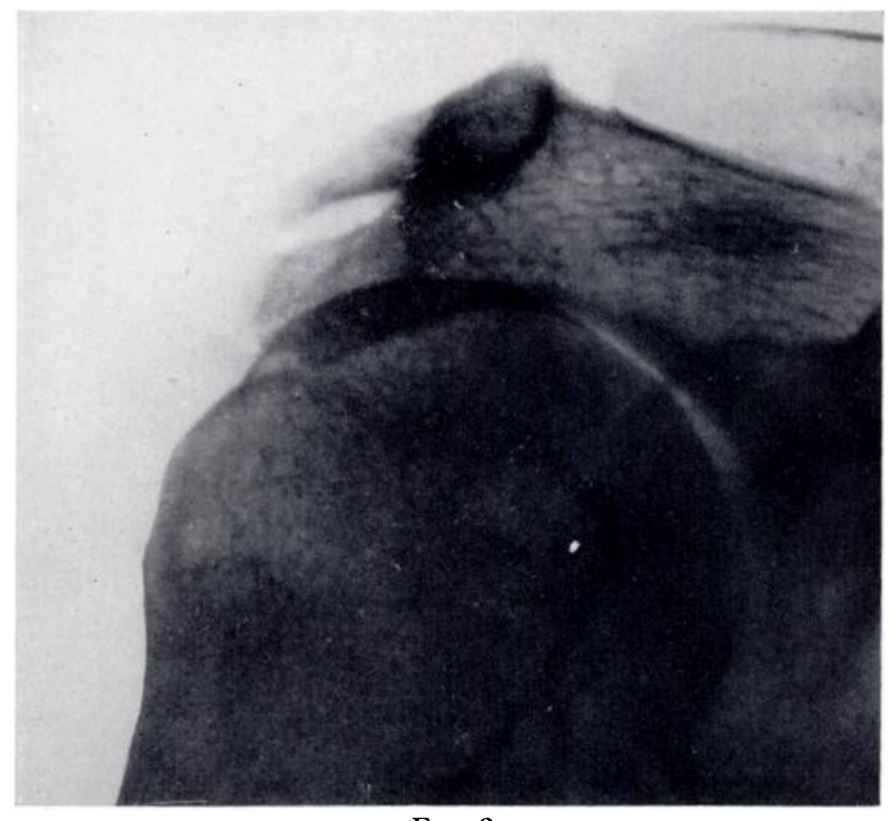

FIG. 2

After excision of the acromion four and a half vears previously the bone has reformed almost completely.

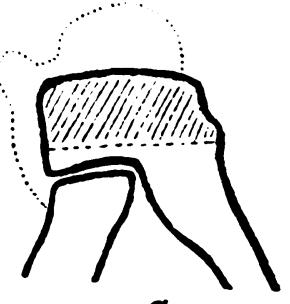

$\boldsymbol{\alpha}$

FiG. 3

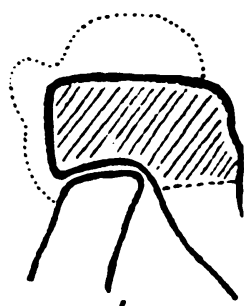

b

Excision of bone distal to the acromio-clavicular joint (Fig. 3a) is not always satisfactory. It is better to remove the acromion completely

(Fig. $3 b$ ) because otherwise new bone may form in the attachment of the deltoid to the raw bone surface.
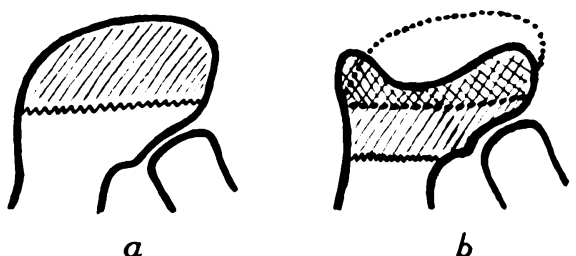

Fig. 4

Fig. $4 a$ is a tracing of the line of first excision of the acromion in the case shown in Fig. 2. New bone that formed within a few months (Fig. 4b) necessitated a second operation for complete excision at the joint level.

acromion process and the insertion of the supraspinatus tendon comes into contact with the anterior part of the process on shoulder movement. In fact Codman suggested that it was contact with the coraco-acromial ligament rather than with bone that caused the pressure. In any event it is essential that the anterior part of the acromion should be removed completely and any attempt to preserve the acromio-clavicular joint may cause unsatisfactory results. Moreover, when the acromion has been divided and the detached deltoid muscle is sutured to its cut edge, new bone formation takes place rapidly and symptoms may recur (Figs. 2-4). If the whole of the acromion process lateral to the acromio-clavicular joint is 


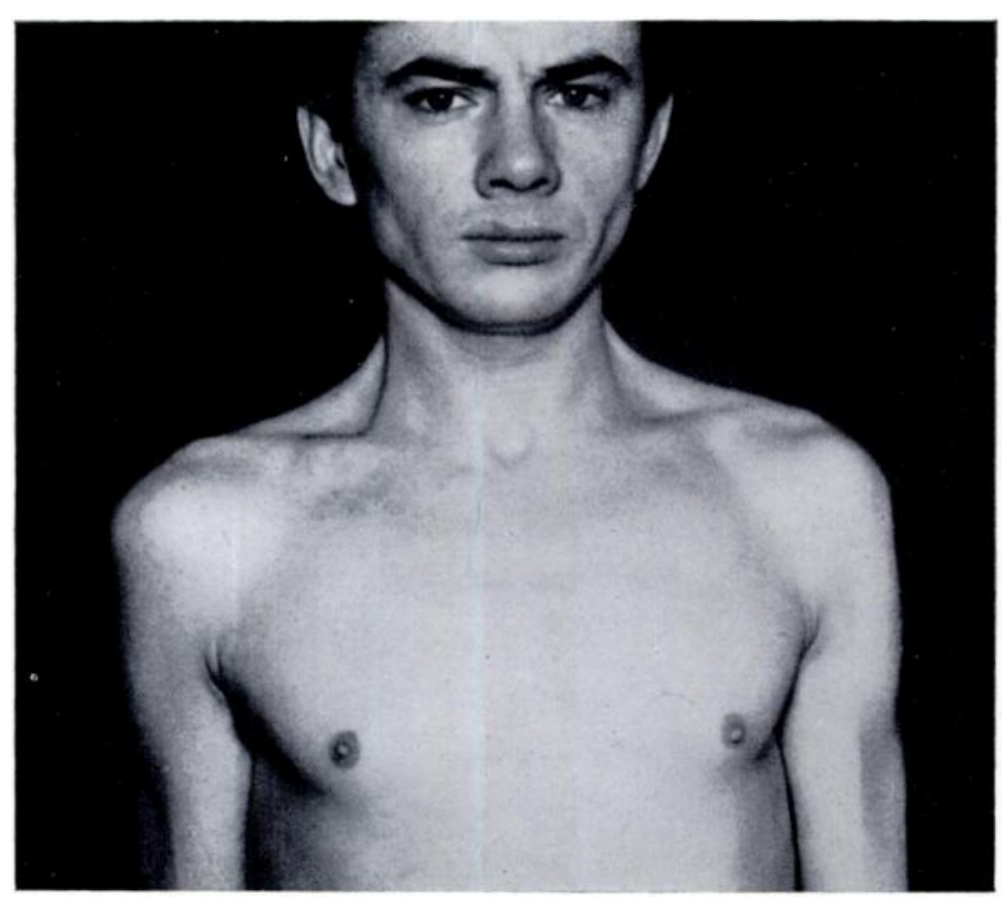

FIG. 5

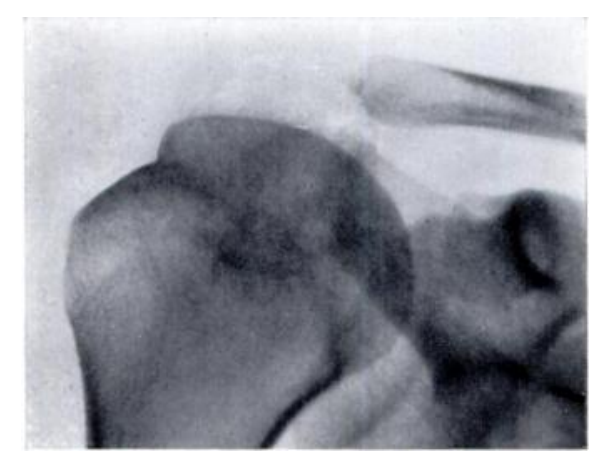

FIG. 6

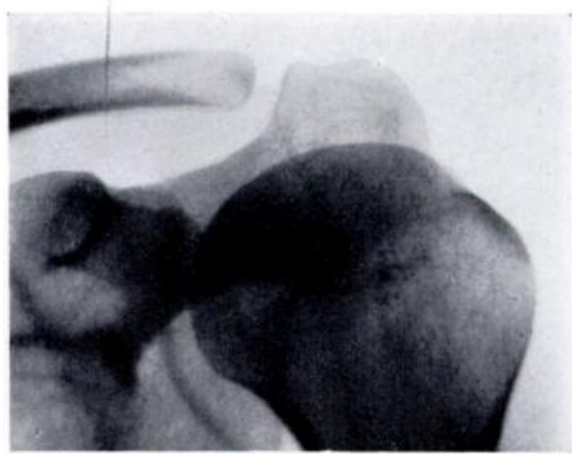

Fig. 7

Photograph of patient four months after complete excision of the right acromion (Fig. 5) and radiograph of the right shoulder (Fig. 6) and the left shoulder (Fig. 7). The difference in contour of the shoulder is scarcely noticeable.

removed, the end of the clavicle constitutes the new attachment to which the deltoid is sutured, so that no raw bone is exposed in the area in which new bone formation would be harmful.

Complete excision of the acromion does not appear to cause untoward effects. If the conoid and trapezoid ligaments are undamaged, loss of the acromio-clavicular joint is not associated with any disability. The cut edge of the deltoid muscle, sutured firmly to the coraco-acromial ligaments, forms a firm fibrous scar which gives support to the outer end of the clavicle. The operation does not result in any serious cosmetic blemish. The scar is of course visible and may tend to stretch a little, but the alteration of contour is not noticeable (Figs. 5 and 6). The posterior angle of the cut edge of bone should be rounded off so that no spike is left. 


\section{INDICATIONS FOR EXGISION OF THE ACROMION}

Broadly speaking, excision of the acromion is indicated in treatment of the supraspinatus syndrome whenever conservative treatment has failed. More precise indications vary according to individual opinion and they depend upon a number of circumstances. The most important single factor is the duration of symptoms. Lesions of the supraspinatus group tend to resolve and subside with time, and operation is never a matter of urgency. Indeed some surgeons maintain that all these lesions recover spontaneously over a period of one or two years; but even if this were true, which is to be doubted, few patients are prepared to tolerate symptoms for so long a time, nor is it reasonable to expect them to do so. On the other hand it is impossible to tell in the early stages which patient will recover and which will require operation. Conservative treatment should always be tried for at least two months; and only if there is no improvement at the end of that time should operation be considered.

When, as is often the case, symptoms improve to some extent without being completely relieved, other factors must determine whether or not operation should be advised. The severity of the symptoms and the degree of associated disability are obviously important. In its initial stages the acute syndrome may be acutely painful and crippling, but this phase does not often persist. The typical syndrome is neither very painful nor completely incapacitating and its effects often depend on the age, occupation and mode of life of the patient. A painful arc of movement may be no more than a mild nuisance to an elderly patient of sedentary habits and yet be a severe handicap to a younger person whose occupations are strenuous. Discomfort, accepted philosophically by one with a phlegmatic temperament, may be devastating in its effects on a more highly strung patient. Each patient who does not respond to conservative treatment provides an individual problem; and the surgeon's attitude to operation will certainly be influenced profoundly by his opinion of its efficacy.

There are two circumstances in which excision of the acromion is contra-indicated. When there is true limitation of shoulder movement, operation produces a stiff and stubborn joint which requires months of treatment before mobility is restored. Muscle spasm can be distinguished from adhesion formation by examination after the lesion has been infiltrated with local anaesthetic or, better still, by examination under a general anaesthetic. If there is true limitation this must be dealt with by active exercises, and perhaps manipulation, before operation is contemplated. Operation is also contra-indicated if there is doubt as to the diagnosis. Removal of the acromion will relieve pressure on the supraspinatus tendon or subacromial bursa but if applied in a haphazard manner to the treatment of all stiff painful shoulders the operation gives very unsatisfactory results, particularly in patients with periarthritis.

\section{RESULTS}

In 1939, Watson-Jones first reported excision of the acromion in the treatment of supraspinatus tendon lesions at a meeting of the British Orthopaedic Association in Oswestry; and he described the procedure in 1943. At about that time this writer was becoming increasingly dissatisfied with the results of conservative treatment in many service patients who were under treatment at the Royal Air Force Hospital, Rauceby. Those who were not promptly relieved by conservative treatment were often incapacitated for long periods and there seemed nothing else to offer. After trial of excision of the acromion the results were encouraging, particularly when experience had shown that it was necessary to excise the whole of the acromion. Conviction that the operation was a good one was strengthened by personal experience. In 1944, after several months of typical incapacity which had persisted unchanged despite all forms of conservative treatment, my own acromion process was excised by Sir Reginald Watson-Jones with complete and permanent cure. The results in a personal series 
of ninety-five patients are summarised in Table I. Because many of these patients were referred for operation after conservative treatment elsewhere had failed, it is not possible to state the proportion that they represent of all patients suffering from the supraspinatus syndrome.

In eighty of ninety-five patients the operation was completely successful; they were relieved of symptoms and regained a full range of powerful shoulder movement. In the first nine operations the acromion was divided far enough laterally to preserve the acromioclavicular joint. Five of these patients gained incomplete relief or none at all. In three patients further excision of bone gave good results. Two re-excisions were carried out about two months after the original operation; the third patient gained initial relief from the first operation but symptoms then recurred and at the time of re-excision seven months later new bone formation was obvious. In the other two unsuccessful cases permission for further operation was refused; one patient was satisfied with the incomplete relief he had gained; the other was discouraged by the unchanged persistence of his original symptoms. In six instances the result of operation was unsatisfactory because there was post-operative

TABLE I

Results of Operation

\begin{tabular}{|c|c|}
\hline \multicolumn{2}{|c|}{$\begin{array}{l}\text { Results of Excision of the Acromion in Ninety-five Patients } \\
\text { SUffering from the Supraspinatus Group of Lesions }\end{array}$} \\
\hline Satisfactory to patient and surgeon & $80(84 \cdot 2$ per cent. $)$ \\
\hline Unsatisfactory & $15(15 \cdot 8$ per cent.) \\
\hline \multicolumn{2}{|c|}{ ANalysis of Fifteen Unsatisfactory Results } \\
\hline Insufficient bone removed & $\begin{array}{l}5 \\
\text { (In three patients a further } \\
\text { excision was successful) }\end{array}$ \\
\hline Post-operative limitation of movement & 6 \\
\hline No relief of symptoms & 4 \\
\hline
\end{tabular}

limitation of shoulder movement; there was complete relief of pain but the range of forward flexion and abduction movement was reduced by about one-third and the patients, being content with relief of pain, were unwilling to be stimulated in making the endeavour needed to regain a normal range of movement. Four patients said that they had gained no relief at all: in two the original diagnosis may have been inaccurate; and in the other two failure of the operation is still unexplained.

\section{PATHOLOGICAL FINDINGS AT OPERATION}

The subacromial bursa was opened at operation in every case and the pathological findings were noted. The various conditions that were seen were not always distinctively different, one from the other. Nevertheless it seemed possible to classify them into four groups (Table II).

Fifty-six patients appeared to have tendinitis with an associated bursitis. The tendon was red, thickened and rough; and the bursal walls and synovial lining were oedematous 
and inflamed, these changes being most marked around the tendon. In recent and acute cases the synovial membrane hung in red, oedematous folds. The changes suggested that tendinitis was the primary lesion and bursitis secondary.

In ten patients the primary lesion appeared to be subacromial bursitis. The walls of the bursa were red, thickened and adherent to the acromion; the cavity contained free fluid and, in a few instances, small loose bodies. The changes were uniform, and not in any way localised to the region of the supraspinatus tendon, and they were exactly similar to those of a chronically inflamed prepatellar or olecranon bursa.

In fifteen cases calcified deposits in the supraspinatus tendon were visible in the preoperative radiographs. At operation the tendon appeared rough, thickened, opaque and slightly red, and there was evidence of localised bursitis. No attempt was made to remove the deposits from the tendon.

Tears of the supraspinatus tendon were found in fourteen patients. Most frequently the tendon was incompletely detached from its insertion. The area of detachment was small and not associated with retraction such as occurs after complete rupture. Occasionally small rents were observed in the tendon itself through which the articular cartilage of the humeral head could be seen.

TABLE II

Pathological Findings

\begin{tabular}{|c|c|}
\hline Pathological Findings in Ninety-Five Patients with the \\
Supraspinatus Sydidome \\
\hline $\begin{array}{c}\text { Tendinitis with secondary subacromial } \\
\text { bursitis }\end{array}$ & 56 \\
\hline Primary subacromial bursitis & 10 \\
\hline $\begin{array}{c}\text { Calcification in supraspinatus tendon } \\
\text { Tears or detachments of supraspinatus } \\
\text { tendon }\end{array}$ & 15 \\
\hline
\end{tabular}

\section{OPERATIVE TEGHNIQUE AND POST-OPERATIVE MANAGEMENT}

Certain points in the technique, which make the operation easier, are worthy of note. The patient is laid on the sound side with the head well flexed. The surgeon sits at the top of the table, the arm being controlled by an assistant. The incision begins in front of the acromio-clavicular joint and extends back across the joint and acromion in a direction slightly concave outwards. A flap of skin and subcutaneous tissue is then raised, exposing the upper surface of the acromion and joint. The periosteum is divided about half an inch lateral to the proposed line of section and reflected inward. The acromion is divided from before backwards with a sharp osteotome held very obliquely to avoid the possibility of damage to underlying structures. The line of section should extend directly backwards from the acromio-clavicular joint. When the acromion has been divided it is held in lion forceps while the deltoid origin is detached from its outer edge, working from behind forwards. The subacromial bursa is usually found to be adherent and must be dissected from the deep surface of the bone. The last structures to be divided are the acromio-clavicular and coracoacromial ligaments.

vol. 31 B, No. 3, August 1949 
After removal of the acromion the bursa is opened for examination, and the underlying tendon is inspected, the arm being abducted and rotated as necessary. The bursal wall is closed. The cut edge of the deltoid is sutured firmly to the acromio-clavicular ligaments and reflected periosteum or, if necessary, to the bone itself through holes drilled with an awl. The deltoid is repaired while the limb is held in abduction and security of the suture-line is then tested by lowering the arm to the side. The skin is sutured and a pressure bandage applied.

During operation some generalised arteriolar bleeding occurs, but no large vessels are encountered and few ligatures are necessary. It is very much easier and quicker to remove the acromion in this retrograde manner than to attempt to clear its muscle attachments and then to divide the bone. The acromion should always be cut cleanly with a sharp osteotome and never be nibbled away piecemeal.

Post-operative management-After operation it is unnecessary to immobilise the limb in abduction. The patient wears a sling but is encouraged to use the forearm and hand as much as possible. Passive movements of the shoulder are permitted, the arm being placed in the most comfortable position or rested on a pillow. The patient need be confined to bed only for four or five days. Active movements of the shoulder are not attempted for ten days after operation during which time contraction of the deltoid is painful; but after this interval gentle shoulder exercises should be encouraged, particular attention being paid to abduction. In the early stages exercises are best tolerated in the supine position which minimises the effects of gravity, and it is advisable to continue abduction exercises in this position until a full range has been regained.

There is of course much variation in the rate of recovery of different patients. An active man may play golf without difficulty within four weeks of operation; but most patients need six to eight weeks before they are able to use the shoulder with confidence. The last ten degrees of abduction and forward flexion movement are regained slowly, and very often, since the patient may not appreciate that there is still some limitation of movement, it is difficult to persuade him to persist assiduously with the necessary exercises.

Acute symptoms are at once relieved by the operation but often there is slight aching, especially at night, for several months. The cure may be regarded as complete when the patient has not only regained a full range of movement but is also able to sleep comfortably at night on the affected limb.

\section{CONCLUSIONS}

1. The supraspinatus group of lesions constitutes one of the two common causes of the painful shoulder.

2. Most, but not all, of these lesions resolve either spontaneously or after conservative treatment.

3. When conservative treatment fails symptoms can be relieved by excision of the acromion process, provided that sufficient bone is removed to relieve all pressure on the tendon throughout a full range of shoulder movement.

4. Excision of the acromion is contra-indicated if there is doubt as to the diagnosis or if there is true limitation of shoulder movement.

\section{REFERENCES}

Watson-Jones, R. (1939): “Excision of acromion for supraspinatus tendinitis." Demonstration of cases at meeting of British Orthopaedic Association, Oswestry.

Watson-Jones, R. (1943) : Fractures and Joint Injuries. Third edition. 418. Edinburgh: E. \& S. Livingstone, Ltd. 